\title{
Patient-reported outcomes used to personalize Dutch head and neck cancer rehabilitation
}

\author{
Lydia FJ van Overveld ${ }^{1 *}$, Lisa MC van Hoogstraten ${ }^{1}$, Robert P Takes ${ }^{2}$, Jozé CC Braspenning¹, Rob J Baatenburg de Jong ${ }^{3}$, Jan P de Boer ${ }^{4}$, \\ Ludi E Smeele ${ }^{5,6}$, Matthias AW Merkx ${ }^{7,8}$ and Rosella PMG Hermens ${ }^{1}$ \\ ${ }^{1}$ Radboud University Medical Center, Radboud Institute for Health Sciences, Scientific Center for Quality of Healthcare, The Netherlands \\ ${ }^{2}$ Department of Otolaryngology, Head and Neck Surgery, Radboud University Medical Center, Radboud Institute for Health Sciences, The Netherlands \\ ${ }^{3}$ Department of Otorhinolaryngology, Head and Neck Surgery, Erasmus MC Cancer Institute, The Netherlands \\ ${ }^{4}$ Department of Medical Oncology, Antoni van Leeuwenhoek Nederlands Kanker Instituut, The Netherlands \\ ${ }^{5}$ Department of Oral and Maxillofacial Surgery, Academisch Medisch Centrum, The Netherlands \\ ${ }^{6}$ Department of Head and Neck Surgery and Oncology, Antoni van Leeuwenhoek Nederlands Kanker Instituut, The Netherlands \\ ${ }^{7}$ Department of Oral and Maxillofacial Surgery, Radboud University Medical Centre, Radboud Institute for Health Sciences, The Netherlands \\ ${ }^{8}$ The Netherlands Comprehensive Cancer Organisation, Utrecht, The Netherlands
}

\begin{abstract}
Objective: Monitoring outcomes of head \& neck cancer (HNC) patients during and after treatment, gives healthcare professionals tools to improve their care and to inform patients in perspective of expectation-management. Since outcomes could differ depending on type of treatment, patient-reported outcomes (PROs) for HNC patients receiving a single-modality treatment versus multi-modality treatment were monitored in order to provide a personalized follow-up.

Study design: Prospective, observational, multicentre study.

Setting: Patients from three hospitals participating in the Dutch Head \& Neck Audit (DHNA).

Methods: Validated PRO measures (PROMs), including Global Health Status (GHS), functioning and symptoms, were completed at baseline and 3, 6, and 12 months after the last treatment. Descriptive analyses were used to assess trends in PROs over time and between treatments.

Results: A total of 358 patients completed 571 PROMs. Global health status tended to score higher in the surgery group (single-modality treatment). Patients with multi-modality treatment tended to have poorer functioning than patients with single-modality treatment (surgery of radiotherapy). Patients who underwent surgery as single-modality treatment tended to have fewer symptoms than patients who received radiotherapy as single-modality or multi-modality treatment.

Conclusions: PROMs are useful tools to personalize HNC care. Given the greater incidence of symptoms and loss of functioning among patients after multimodality treatment, follow-up care has to be improved by introducing a more personalised information (preventive expectation management and supportive care advice) at the start of a multi-modality treatment and follow-up phase directed toward rehabilitation for restoring function and decreasing symptoms.
\end{abstract}

\section{Introduction}

'In health care, as in other areas, rapid change can be stimulated by crises. With the medical crises of the COVID-19 pandemic and our imperative to provide high-quality care to our patients with cancer, a rapid implementation of web based PROs in clinical practice is a critical change to assist oncologists and patients' [1]. Patient-reported outcomes (PROs) measured with patient-reported outcome measures (PROMs) provide information about the patients' feelings during the healthcare process. This information can be used as (1) feedback as a starting point for healthcare improvement [2], (2) an opportunity to inform patients of their own health-status during treatment and followup, and (3) a tool to personalise the individual healthcare process especially in times when intermediate control visits are avoided, for example during the COVID-19 pandemic [3,4]. Web-based PROs have been shown to improve quality of life (QoL), decrease emergency clinic visits, and improve performance status and the number of patients receiving active cancer treatments at disease progression [5]. Basch and colleagues even state that integration of PROs into routine care for patients with metastatic cancer was associated with increased survival compared to usual care [4]. One explanation for the great effects is that reviewing PROMs with cancer patients increases discussion of symptoms such as pain and emotional distress between the physician and the patient [6].

PROs are mainly used to evaluate treatment effects. Their use in follow-up care may have the potential to shift healthcare delivery toward a more patient-centred approach [7]. During treatment, PROs are primarily used to illuminate the dialogue about symptom management.

${ }^{*}$ Correspondence to: Lydia FJ van Overveld, Radboud University Medical Center, Scientific Center for Quality of Healthcare (114), PO Box 9101, $6500 \mathrm{HB}$ Nijmegen, The Netherlands, Tel: +3124-3616538; E-mail: Lydia.vanOverveld@ radboudumc.nl

Key words: head and neck cancer care, patient-reported outcomes, personalized care

Received: May 08, 2020; Accepted: May 21, 2020; Published: May 25, 2020 
In follow-up care, cancer patients experience a more varying set of needs, but with fewer opportunities to report problems to the clinician. Here, the potential of a screening tool to detect patients' individual needs may be helpful to support personalized rehabilitation [7].

A prime example of the need of high-quality oncologic care is the delivery of care to patients with HNC. HNCs on itself and their treatment have a significant impact on the patient's well-being because they grow relatively quickly in an anatomically and functionally complex area [8-10]. Patients often experience problems with speech, swallowing, and physical disfiguration due to treatment [11-13]. The treatment may consist of surgery or radiotherapy as single-modality treatment or in combination e.g. with systemic therapy as a multimodality treatment. Optimal alignment and cooperation of medical specialists, allied healthcare professionals and the individual patients from the perspective of expectation-management during and after treatment, is crucial for an optimal result.

In the Dutch Head \& Neck Audit (DHNA), PROs were included as outcome indicators and were considered to be the standard assessment to experience their disease, treatment and revalidation [14]. We aimed to get insight into the trends and differences between single-modality treatment and multi-modality treatment for HNC patients on different follow-up moments to obtain information about specific aspects of different treatment methods to be used for personalizing follow-up care and cure.

\section{Methods \\ Study design}

In a prospective, observational, multicentre study, patients diagnosed with a primary HNC tumour with a curative intent of treatment were asked to participate. Two different validated PROMs were used: The European Organization for Research and Treatment of Cancer Quality-of-Life Questionnaire (EORTC QLQ-C30) and the EORTC Head and Neck Cancer Module (H\&N35) [15-17]. Both questionnaires have been exclusively tested in several populations, as well as in several languages and cultural settings [15-17].

We determined the differences and trends between single-modality treatment and multi-modality treatment on different follow-up moments to obtain information about specific aspects of different treatment methods to be used for personalizing follow-up care and cure.

\section{Study population}

Patients with a primary HNC were asked to fill in the questionnaires to measure PROs and PREs at pre-defined moments (see data collection) (Table 1).

\section{Data collection}

The EORTC-QLQ-C30 and the H\&N35 were digitally completed at baseline and 3, 6, and 12 months of follow-up after the last treatment (Figure 1). 'Baseline' was defined as the date of diagnosis during multidisciplinary team meetings. The follow-up moments were aligned with other national initiatives.

An online self-registration system was used by health professionals to register and analyse data and patient characteristics. The questionnaires were sent automatically to the patients at pre-defined moments by a specific application in the online self-registration system (ePROMs). All data for PROMs, and patient characteristics registered in the online self-registration system were collected in a pseudonymised database.
Table 1. Definition of head and neck cancer. Only malignant tumours of the salivary glands and squamous cell carcinomas were included.

\begin{tabular}{|l|l|}
\hline Definition of head and neck cancer & ICD-O ${ }^{\mathbf{1}}$ codes \\
\hline Oral cavity & $\mathrm{C} 01-\mathrm{C} 06$ \\
\hline Oropharynx & $\mathrm{C} 09, \mathrm{C} 10$, and $\mathrm{C} 14$ \\
\hline Nasopharynx & $\mathrm{C} 11$ \\
\hline Hypopharynx & $\mathrm{C} 12$ and $\mathrm{C} 13$ \\
\hline Larynx & $\mathrm{C} 32$ \\
\hline Nasal cavity and paranasal sinuses & $\mathrm{C} 30$ and C31 \\
\hline Malignant salivary glands & $\mathrm{C} 06.9, \mathrm{C} 07$, and $\mathrm{C} 08$ \\
\hline Lymph node squamous cell carcinoma of unknown origin & $\mathrm{C} 80$ \\
\hline
\end{tabular}

${ }^{1} \mathrm{ICD}-\mathrm{O}=$ International Classification of Diseases for Oncology

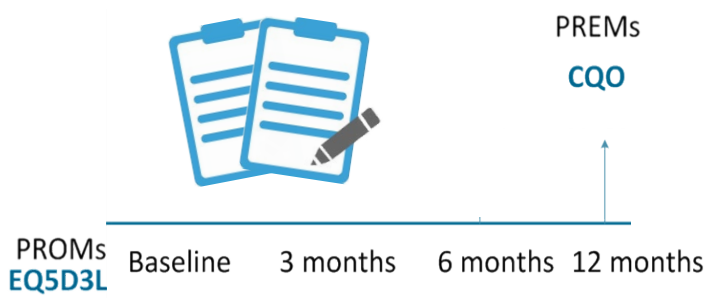

EORTC QLQ-C30

EORTC QLQ-H\&N35
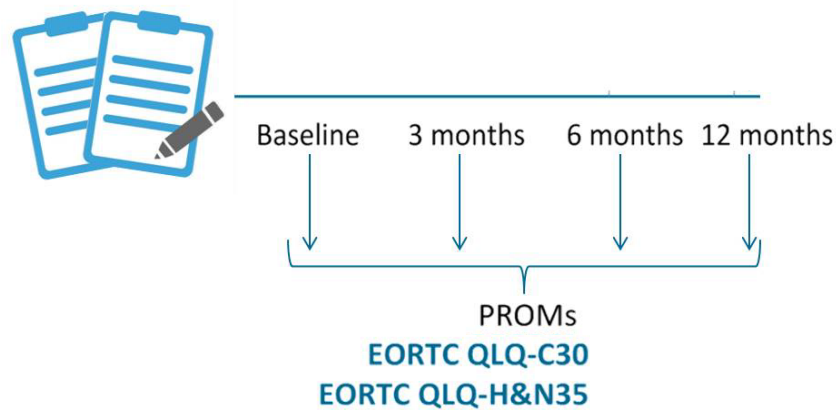

Figure 1. Administration of the PROMs at pre-defined moments

We evaluated the forms filled in between November 2014 and February 2017.

\section{Data analyses}

All the domains of the PROMs were included in the analyses. All variables were transformed to scales from 0 to 100 and divided into scale scores according to the EORTC QLQ-C30 rules and the H\&N35 Scoring Manual [18]. Because of the expected low numbers of patients per treatment group at each follow-up moment, specific trends of the various follow-up moments were observationally assessed whether these trends differed between single-modality treatment and multimodality treatment on the different follow-up moments. Singlemodality treatments were split into surgery alone and radiotherapy alone since quality-of-life aspects could differ depending on which treatment the patient received. Clinical relevance was set at a difference of 10 or more points on a scale of 0 to 100 in the EORTC symptom scores $[11,19]$.

\section{Ethics}

The Medical Ethical Committee of the region Arnhem-Nijmegen declared that ethical approval was not necessary $(2014 / 070)$. 


\section{Results}

\section{Study population}

Of all patients included in the DHNA, a total of 358 patients completed 571 questionnaires containing both the EORTC QLQ-C30 and EORTC QLQ-H\&N35 at different follow-up moments (Table 2). The different patient groups had a mean age between 59 and 68 (Table 3). Most patients who filled in a PROM (48\%) received a multi-modality treatment (surgery + radiotherapy \pm chemotherapy); $28 \%$ received only radiotherapy and $24 \%$ received only surgery as single therapy.

\section{Patient-reported outcomes}

\section{Global health status}

The global health status (GHS) tended to increase with a clinically relevant differences (10 or more points) from baseline to 12 months after follow-up for surgery, radiotherapy, and multi-modality therapy (Figure 2). In the single-modality surgery group, the GHS had already had a major increase of 9.5 points at 3 months after the last treatment compared to multi-modality treatment and single-modality treatment radiotherapy ( 3.1 and -0.4 points respectively).

\section{Functioning}

A higher score on the function scales means better functioning. Overall, surgery as single-modality treatment seemed to score better on

Table 2. Number of patients with different treatments that completed the PROMs at different follow-up moments

\begin{tabular}{|l|c|c|c|c|}
\hline & Baseline & $\mathbf{3}$ & $\mathbf{6}$ & $\mathbf{1 2}$ \\
\hline PROMs - Surgery & 41 & 15 & 19 & 10 \\
\hline PROMs - Radiotherapy & 35 & 23 & 29 & 13 \\
\hline PROMs - Multimodality & 52 & 48 & 46 & 27 \\
\hline
\end{tabular}

functioning for most functional scales. In other words, the red line is often the upper line. However, radiotherapy seemed to overtake the red line after 6 months follow-up on the domain's role functioning, physical functioning, and social functioning. After 6 months, all functional scales seemed to increase after radiotherapy as single-modality treatment where surgery or multi-modality treatments seemed to increase less steep or even decrease after 6 months (Figure 2).

\section{Symptoms}

A higher score on the symptom scales means more problems for the patient. We distinguished three types of trends: a similar trend, a peak at 3 months after the last treatment, and varying trends (Figure 3 ). The symptom Pain tended to decrease with clinical relevance difference up to 12 months after follow-up for all treatment types. The symptom Nausea vomiting tended to remain horizontal for all treatment types (Figure 3A). The symptoms Sticky saliva and Problems of the senses each showed a clear peak at 3 months after the last treatment, which means that more problems occur (Figure 3B). All symptoms in both $3 \mathrm{~A}$ and $3 \mathrm{~B}$ showed clearly that a multi-modality treatment seemed to give rise to the most problems, followed by radiotherapy as singlemodality treatments. The lesser problems are caused by surgery as single-modality treatment. The symptoms Less sexuality and Insomnia showed no clear trend that was similar for the type of treatment (Figure 3C). However, clinically relevant differences were seen over different follow moments for different treatments. A summary of results is given in figure 4.

\section{Discussion}

We assessed trends and differences between single-modality treatment and multi-modality treatment to obtain information about specific aspects of different treatment methods in order to personalize HNC care on different follow-up moments. The PROs showed that global

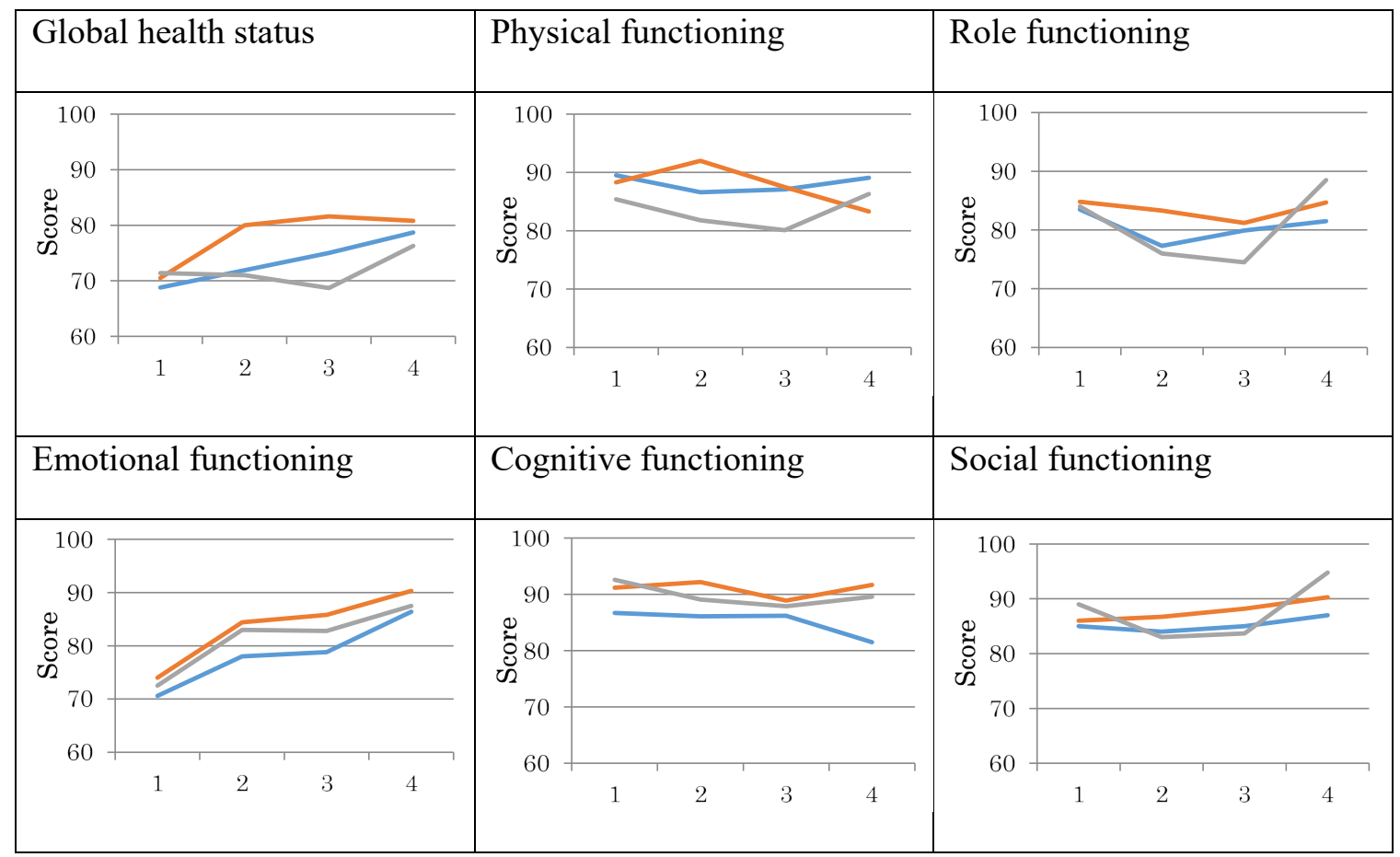

Figure 2. Global health status and functioning scales from the EORTC-C30 for patients with head and neck cancer. Global health status and functioning scales from the EORTC-C30 for patients with head and neck cancer. Red, surgery; green, radiotherapy; blue, multi-modality treatment; X axis: 1, baseline; 2, 3 months after last treatment; 3 , 6 months after last treatment; 4, 12 months after last treatment; Y axis: EORTC-C30 score (0 to 100). 


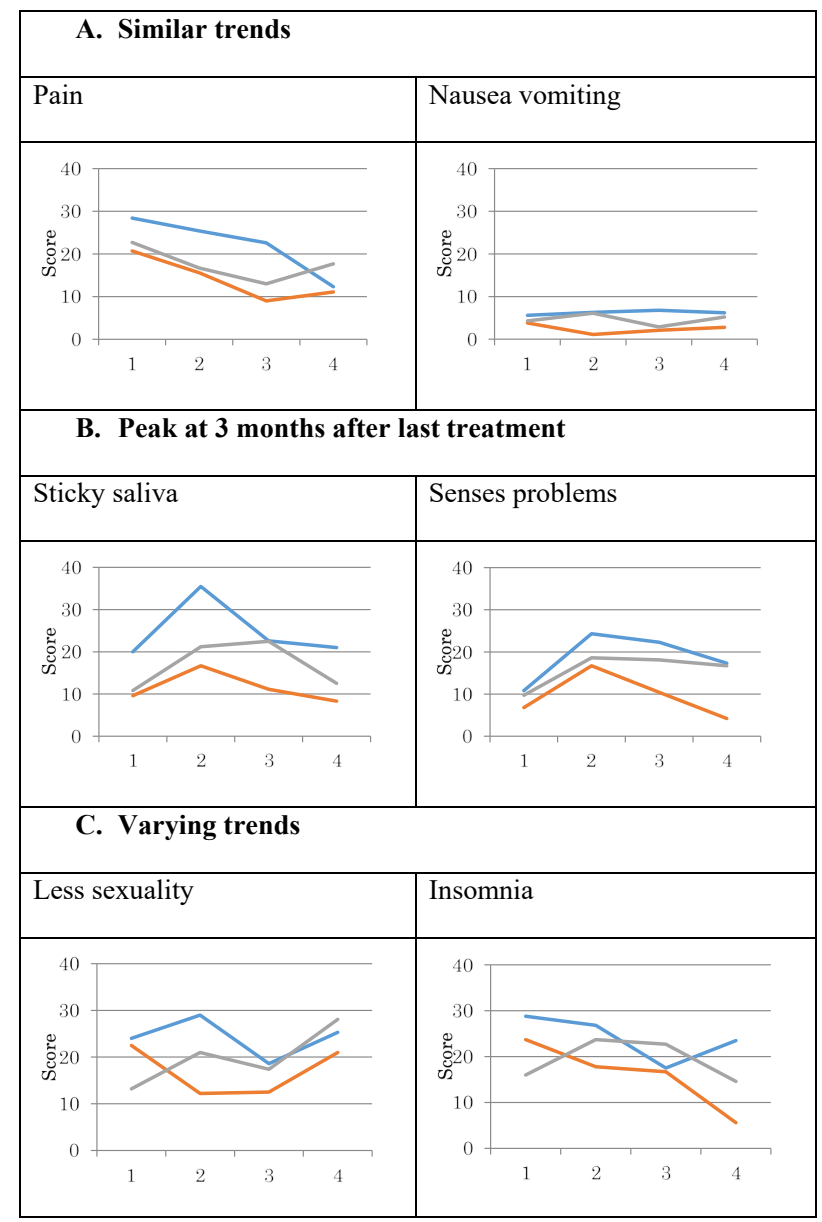

Figure 3. Examples of trends in EORTC-C30 and EORTC-H\&N35 of patients with head and neck cancer. Examples of trends in EORTC-C30 and EORTC-H\&N35 of patients with head and neck cancer. Red, surgery; green, radiotherapy; blue, multi-modality treatment; X axis: 1, baseline; 2, 3 months after last treatment; 3,6 months after last treatment; 4 , 12 months after last treatment; Y axis: EORTC-C30 and EORTC-H\&N35 (0-100).

General

A higher score on the function scales means better functioning and a higher score on the symptom scales means more problems for the patient.

- Functioning and symptoms seemed to differ between follow-up moments and type of treatment

Patients who received radiotherapy as single-modality treatment tends to have better functioning and fewer symptoms than patients who received radiotherapy as multi-modality treatment

- Patients who underwent surgery as mono-therapy tended to have fewer symptoms than patients who received radiotherapy as single-modality treatment or a multi-modality.

- Up to 6 months follow-up, a treatment with surgery tends to have better functioning compared to radiotherapy as singlemodality treatment. Radiotherapy as single-modality treatment tends to give better functioning after 6 months follow-up.

Global health status

- Global health status in surgery group (single treatment) has the steepest increase at 3 months after the last treatment compared to multi-modality treatment and single-modality treatment radiotherapy

Functioning

- Emotional functioning tends to increase with both single and multi-modality treatment

Surgery as single-modality treatment seemed to score better on functioning for most functional scales. However, radiotherapy seemed to overtake after 6 months follow-up on the domains role functioning, physical functioning and social functioning.

\section{Symptoms}

- The symptom Pain tends to decrease with both single and multi-modality treatment

Most symptoms show that a multi-modality treatment give the most problems, followed by radiotherapy as singlemodality treatments, up to 12 months after last treatment.

Better functioning (up to 6

months follow-up)*:

1. Surgery (single)

2. Radiotherapy (single)

3. Multi-modality treatment
Better functioning (after 6
months follow-up)*:
1. Radiotherapy (single)
2. $\quad$ Surgery (single)
3. $\quad$ Multi-modality treatment

\author{
Less symptoms*: \\ 1. Surgery (single) \\ 2. Radiotherapy (single) \\ 3. Multi-modality treatment
}

* 1 is the best functioning or the least symptoms, 3 is the worst functioning or the most symptoms.

Figure 4. Summary of results for both functioning and symptoms. 
Table 3. Characteristics of patients who completed the PROMs.

\begin{tabular}{|c|c|c|c|}
\hline & \multicolumn{3}{|c|}{ PROMs } \\
\hline & \multicolumn{2}{|c|}{ Single-modality } & \multirow{2}{*}{\begin{tabular}{|c} 
Multi-modality \\
$\mathbf{( \% )}$ \\
$n=173$
\end{tabular}} \\
\hline & $\begin{array}{c}(\%) \\
\text { Surgery } \\
n=85\end{array}$ & $\begin{array}{c}\mathbf{R A T}^{\mathbf{a}} \\
n=100\end{array}$ & \\
\hline Median age at start of treatment & 67 years & 66 years & 66 years \\
\hline \multicolumn{4}{|l|}{ Gender } \\
\hline Male & 78 & 78 & 73 \\
\hline Female & 22 & 22 & 27 \\
\hline \multicolumn{4}{|l|}{ Tobacco smoking } \\
\hline Never smoker & 13 & 9 & 18 \\
\hline Former smoker & 35 & 26 & 40 \\
\hline Current smoker & 21 & 37 & 28 \\
\hline Data missing & 31 & 28 & 14 \\
\hline \multicolumn{4}{|l|}{ Alcohol use } \\
\hline Never drinker & 18 & 16 & 16 \\
\hline Former drinker & 5 & 3 & 8 \\
\hline Current drinker & 60 & 60 & 63 \\
\hline Data missing & 17 & 21 & 13 \\
\hline \multicolumn{4}{|l|}{ Performance status } \\
\hline None & 4 & 4 & 3 \\
\hline Performance status registered & 55 & 48 & 62 \\
\hline 1 Score 0 (perfect health) & 40 & 40 & 53 \\
\hline 2 Score $>0$ & 15 & 8 & 8 \\
\hline 3 Data missing & 45 & 52 & 38 \\
\hline Data missing & 35 & 48 & 35 \\
\hline \multicolumn{4}{|l|}{ Tumour site } \\
\hline Other & 15 & 13 & 27 \\
\hline Oral cavity and oropharynx & 44 & 27 & 47 \\
\hline Larynx and hypopharynx & 37 & 58 & 21 \\
\hline Data missing & 5 & 6 & 4 \\
\hline \multicolumn{4}{|l|}{ Clinical TNM stage ${ }^{b}$} \\
\hline Early (Stage $\leq 2$ ) & 63 & 42 & 18 \\
\hline Advanced (Stage >2) & 12 & 38 & 64 \\
\hline Data missing & 24 & 21 & 18 \\
\hline \multicolumn{4}{|l|}{ Comorbidity } \\
\hline Score 0 & 31 & 37 & 21 \\
\hline Score 2 & & 1 & \\
\hline Score 3 & 9 & 11 & 23 \\
\hline Data missing & 61 & 51 & 57 \\
\hline
\end{tabular}

a) $\mathrm{RAT}=$ Radiotherapy; ${ }^{\text {b) }} \mathrm{TNM}=$ tumour nodes metastasis.

health status, functioning and symptoms seemed to differ between follow-up moments and between type of treatment. In general, patients who received radiotherapy as single-modality treatment tended to have better functioning and fewer symptoms than patients who received radiotherapy as multi-modality treatment. In addition, patients who underwent surgery as single-modality treatment tended to have fewer symptoms than patients who received multi-modality or radiotherapy as single-modality treatment. Furthermore, up to 6 months follow-up a treatment with surgery tended to have better functioning compared to radiotherapy as single-modality treatment. Radiotherapy as singlemodality treatment tended to give better functioning after 6 months follow-up compared to surgery or multi-modality treatment.

As expected from clinical expertise, our study shows that the type of treatment influences how patients feel during the oncologic care process. Certain symptoms are specific to the type of treatment and will therefore always come forward, for example, a dry mouth after radiotherapy. However, for non-specific functions and symptoms, we also expected that multi-modality treatment would give poorer results than single-modality treatment since more alignment, collaboration, and co-ordination is necessary in the healthcare process.

Until now, PROs are mainly used to evaluate treatment effects and their use to inform decisions in follow-up care for individual patients, is relatively rare [7]. Our study gives a prime example of how PROs can be measured in follow-up and of various outcomes from different types of treatment. Unlike other studies, we focussed on type of treatment and different follow-up moments and not on specific patient characteristics or type of tumour $[20,21]$. With only taking the non-specific patient characteristics into account, results are solely based on patient outcomes and not on type of tumour, age or gender. Ramaekers et al. [22] studied the negative association of complications with lower utility and Visual Analog Scale (VAS) score and shows that adding surgery to radiotherapy (multi-modality) decreases the quality of life of HNC patients. In more detail, there are studies that even show that different techniques for radiotherapy or surgery result in different HNC-related symptoms $[20,23]$.

Villaret [23] showed that the quality of life of patients who underwent a reconstruction after being diagnosed with oral cavity cancer decreases dramatically during the first 3 months. Hereafter, it increases. Similar trends were seen in the functioning scales in this study, but not for symptoms. The explanation is that $48 \%$ of the patients also received post-operative radiotherapy or chemo-radiotherapy. In addition, patients completed the questionnaires at follow-up moments after the operation (even though radiotherapy and chemoradiation were given). In our study, patients completed the questionnaires after the last treatment. Probably the patients in Villaret's [23] study had not recovered optimally due to the additional treatments, which adds another dimension to their results.

Our study showed that some functioning and symptom scales present similar trends for both multi-modality or single-modality treatment. Therefore, standardized patient protocols regarding rehabilitation that are adjusted for the type of treatment are useful. However, other functioning and symptom scales show no clear trend for the type of treatment. This underlines the need to personalize the rehabilitation and to use PROMs to adjust the rehabilitation to patient's needs. In the DHNA, the online system can be expanded for health professionals to use the PROs in their consultations.

\section{Strengths and limitations}

We performed an observational study with 358 patients who completed one of more PROMs. There were three reasons for low response: non-response of patients after the first PROM, patients were not able to complete PROMs at each follow-up moment, and patients had not yet reached the time of one year of follow-up after the last treatment. We choose to perform trend analysis because the number of patients for each treatment group at each follow-up moment was sometimes less than 20 . When more data become available in the future, possible differences can be tested for various follow-up moments and different treatments using statistics. Patients in this study were included from three different specialised hospitals. When more data is available, differences between hospitals and the influence of patient characteristics can be analysed.

\section{Conclusion}

In conclusion, a patient who undergoes multi-modality treatment achieves poorer functional scores and has more symptoms than patients who undergo single-modality treatment. Given the greater incidence of 
symptoms and greater loss of function in patients after multi-modality treatment, the follow-up phase should be directed to personalized rehabilitation by restoring function and decreasing symptoms monitored by PROMs. In addition, patients should be informed in the perspective of expectation-management. It is crucial for medical specialists, allied healthcare professionals and the individual patients to be aware that a multi-modality treatment goes along with poorer functioning and more symptoms. Perhaps a medical crisis like the COVID-19 pandemic could indeed stimulate the implementation of web-based PROs in clinical practice to assist oncologists and patients. For both single-modality treatments and multi-modality treatments, monitoring PROs should become standardized in the protocol, userfriendly and self-evident. The results should be more visible for health professionals in order to personalize HNC follow-up.

\section{Funding}

The study sponsor CZ health insurer did not have any role in study design and the collection, analysis, and interpretation of data, nor in writing the article and the decision to submit it for publication; besides the researchers were independent from funders and sponsors.

\section{Reference}

1. Marandino L, Necchi A, Aglietta M, Di Maio M (2020) COVID-19 Emergency and the Need to Speed Up the Adoption of Electronic Patient-Reported Outcomes in Cancer Clinical Practice. JCO Oncol Pract 16: 295-298.

2. van Overveld LFJ, Takes RP, Vijn TW, Braspenning JCC, de Boer JP, et al. (2017) Feedback preferences of patients, professionals and health insurers in integrated head and neck cancer care. Health Expectations 20: 1275-1288. [Crossref]

3. Black N (2013) Patient reported outcome measures could help transform healthcare. $B M J$ 346: f167. [Crossref]

4. Basch E, Deal AM, Dueck AC, Scher HI, Kris MG, et al. (2017) Overall Survival Results of a Trial Assessing Patient-Reported Outcomes for Symptom Monitoring During Routine Cancer Treatment. JAMA 318: 197-198. [Crossref]

5. Livanainen S, Alanko T, Peltola K, Konkola T, Ekström J, et al. (2019) ePROs in the follow-up of cancer patients treated with immune checkpoint inhibitors: a retrospective study. J Cancer Res Clin Oncol 145: 765-774. [Crossref]

6. Stover A, Irwin DE, Chen RC, Chera BS, Mayer DK, et al. (2015) Integrating PatientReported Outcome Measures into Routine Cancer Care: Cancer Patients' and Clinicians' Perceptions of Acceptability and Value. EGEMS (Wash DC) 3: 1169. [Crossref]

7. Riis CL, Bechmann T, Jensen PT, Coulter A, Steffensen KD (2019) Are patientreported outcomes useful in post-treatment follow-up care for women with early breast cancer? A scoping review. Patient Relat Outcome Meas 10: 117-127. [Crossref]

8. Argiris A, Karamouzis MV, Raben D, Ferris RL (2008) Head and neck cancer. Lance 371: 1695-1709.
9. Shiraz F, Rahtz E, Bhui K, Hutchison I, Korszun A (2014) Quality of life, psychological wellbeing and treatment needs of trauma and head and neck cancer patients. Br J Oral Maxillofac Surg 52: 513-517. [Crossref]

10. Gritz ER, Carmack CL, de Moor C, Coscarelli A, et al. (1991) First year after head and neck cancer: quality of life. J Clin Oncol 17: 352-360. [Crossref]

11. Hammerlid E, Wirblad B, Sandin C, Edström S, Kaasa S, et al. (1998) Malnutrition and food intake in relation to quality of life in head and neck cancer patients. Head Neck 20: 540-548. [Crossref]

12. De Boer MF, McCormick LK, Pruyn JF, Ryckman RM, van den Borne BW (1999) Physical and psychosocial correlates of head and neck cancer: a review of the literature. Otolaryngol Head Neck Surg 120: 427-436. [Crossref]

13. van den Berg MG, Rasmussen-Conrad EL, Gwasara GM, Krabbe PF, Naber AH, et al. (2006) A prospective study on weight loss and energy intake in patients with head and neck cancer, during diagnosis, treatment and revalidation. Clin Nutr 25: 765-772. [Crossref]

14. van Overveld LF, Braspenning JC, Hermens RP (2017) Quality indicators of integrated care for patients with head and neck cancer. Clin Otolaryngol 42: 322-329. [Crossref]

15. Sherman AC, Simonton S, Adams DC, Vural E, Owens B, et al. (2000) Assessing quality of life in patients with head and neck cancer: cross-validation of the European Organization for Research and Treatment of Cancer (EORTC) Quality of Life Head and Neck module (QLQ-H\&N35). Arch Otolaryngol Head Neck Surg 126: 459-467. [Crossref]

16. Abendstein H, Nordgren M, Boysen M, Jannert M, Silander E, et al. (2005) Quality of life and head and neck cancer: a 5 year prospective study. Laryngoscope 115: 21832192. [Crossref]

17. Bjordal K, Hammerlid E, Ahlner-Elmqvist M, de Graeff A, Boysen M, et al. (1999) Quality of life in head and neck cancer patients: validation of the European Organization for Research and Treatment of Cancer Quality of Life Questionnaire-H\&N35. Clin Otolaryngol 17: 1008-1019. [Crossref]

18. Rogers SN, Lowe D, Humphris G (2000) Distinct patient groups in oral cancer: a prospective study of perceived health status following primary surgery. Oral Oncol 36: 529-538. [Crossref]

19. McCabe RM, Grutsch JF, Nutakki SB, Braun DP, Markman M (2014) Can quality of life assessments differentiate heterogeneous cancer patients? PLoS One 9: e99445. [Crossref]

20. Wan Leung S, Lee TF, Chien CY, Chao PJ, Tsai WL, et al. (2011) Health-related quality of life in 640 head and neck cancer survivors after radiotherapy using EORTC QLQ-C30 and QLQ-H\&N35 questionnaires. BMC Cancer 11: 128. [Crossref]

21. Dinescu FV, Tiple C, Chirila M, Muresan R, Drugan T, et al. (2016) Evaluation of health-related quality of life with EORTC QLQ-C30 and QLQ-H\&N35 in Romanian laryngeal cancer patients. Eur Arch Otorhinolaryngol 273: 2735-2740. [Crossref]

22. Ramaekers BL, Joore MA, Grutters JP, van den Ende P, de Jong J, et al. (2011) The impact of late treatment-toxicity on generic health-related quality of life in head and neck cancer patients after radiotherapy. Oral Oncol 47: 768-774. [Crossref]

23. Villaret AB, Cappiello J, Piazza C, Pedruzzi B, Nicolai P (2008) Quality of life in patients treated for cancer of the oral cavity requiring reconstruction: a prospective study. Acta Otorhinolaryngol Ital 28: 120-125. [Crossref]

Copyright: (C2020 FJ van Overveld L. This is an open-access article distributed under the terms of the Creative Commons Attribution License, which permits unrestricted use, distribution, and reproduction in any medium, provided the original author and source are credited. 